\title{
Risk Factors in Young Female Patient with Polycystic Ovary Syndrome presenting with Acute Myocardial Infarction
}

\author{
Vivek Vilas Manade ${ }^{1} \quad$ Sridevi C. ${ }^{1}$ \\ 'Department of Cardiology, Dr. D. Y. Patil Medical College, Pimpri, \\ Pune, Maharashtra, India \\ Address for correspondence Vivek Vilas Manade, MD, Department \\ of Cardiology, Dr. D. Y. Patil Medical College, Pimpri, Pune 411018, \\ Maharashtra, India (e-mail: dr.vivek.manade7@gmail.com).
}

Ind J Car Dis Wom:2020;5:327-330

\author{
Abstract \\ Keywords \\ - acute myocardial \\ infarction \\ - low-dose oral \\ contraceptives \\ - young female
}

Polycystic ovary syndrome (PCOS) is known to cause weight gain, nonspecific inflammation, dyslipidemia, and coronary artery disease (CAD).

Oral contraceptives (OCs) also cause venous and arterial thromboembolism, cerebrovascular accidents (CVA), and acute coronary syndrome (ACS). Myocardial infarction (MI) is comparatively less common in patients taking newer generation OCs, particularly if risk factors for CAD are absent, but the risk for venous thromboembolism is still high.

Therefore, OCs should be considered in properly selected patients after weighing risks and benefits.

\section{Introduction}

Polycystic ovary syndrome (PCOS) is associated with acute coronary syndrome (ACS), particularly acute myocardial infarction (AMI) secondary to obesity, low-grade inflammation, dyslipidemia, and endothelial dysfunction. This risk of AMI further increases with the presence of traditional risk factors for MI.

We are presenting a case of a young female on oral contraceptives (OCs) for PCOS with total thrombotic occlusion of left anterior descending (LAD) artery causing AMI with multiple risk factors.

\section{Case Report}

A 22-year-old female residing at Pimpri-Pune, 2nd year M.A. student, presented with chest pain for a period of 8 to 9 hours. The patient was apparently stable in the morning, but then she developed a sudden onset of chest pain while having her lunch at approximately 2:30 PM. The pain was burning in nature, lasting for a few minutes, radiating to the epigastric region, and not being relieved by taking antacids and pain killers. It was not associated with cough, expectoration, dyspnea,

published online December 31, 2020
DOI https://doi.org/ $10.1055 / \mathrm{s}-0040-1722556$ palpitations, syncope, fever, trauma, and nausea or vomiting. The intensity of chest pain increased at approximately 3:00 PM for which she came to Dr. D. Y. Patil Hospital, Pimpri, Pune at about 4:00 pm She was hemodynamically stable. Her ECG on

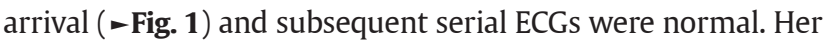
CPK-MB was 28 and Trop-T negative. She was advised hospitalization but she refused to stay.

At about 9:00 PM, she developed severe retrosternal chest pain associated with profuse sweating and giddiness. The pain was radiating to the back, neck, and left arm, and persistent. She was brought by her friends to the hospital at approximately 11:00 PM. Her ECG was showing extensive anterior wall MI (-Fig. 2).

Her ECHO revealed apical, apico-septal, midseptal, and apico-anterior segments were akinetic. EF: $35 \%$

She was K/C/O PCOS for 3 to 4 years and on regular treatment. She was taking tablet DEVIRY $10 \mathrm{mg}$ (medroxyprogesterone) one tablet daily for 3 to 4 years continuously and tablet ORAL-L $(0.15 \mathrm{mg}$ levonorgestrel and $30 \mu \mathrm{g}$ Ethinyl estradiol) since the last 1 month once daily along with some Ayurvedic medications.

C2020. Women in Cardiology and Related Sciences.

This is an open access article published by Thieme under the terms of the Creative Commons Attribution-NonDerivative-NonCommercial-License, permitting copying and reproduction so long as the original work is given appropriate credit. Contents may not be used for commercial purposes, or adapted, remixed, transformed or built upon. (https://creativecommons.org/licenses/by-nc-nd/4.0/).

Thieme Medical and Scientific Publishers Pvt. Ltd. A-12, 2nd Floor, Sector 2, Noida-201301 UP, India 


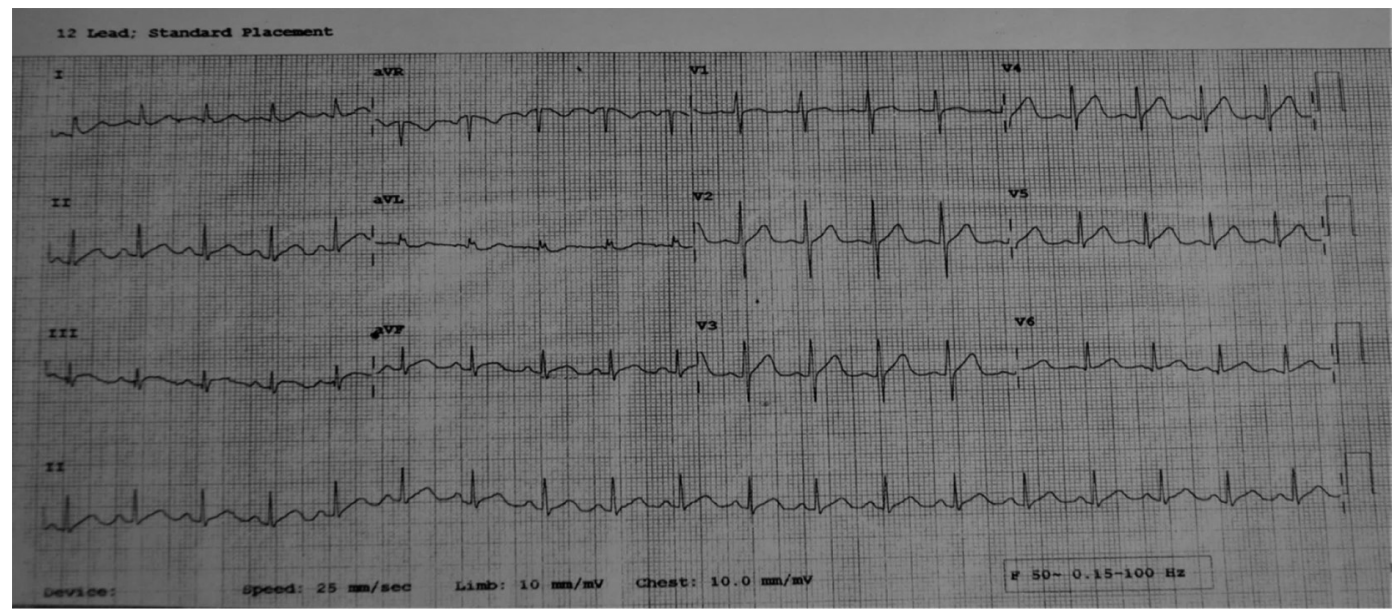

Fig. 1 1st ECG on presentation.

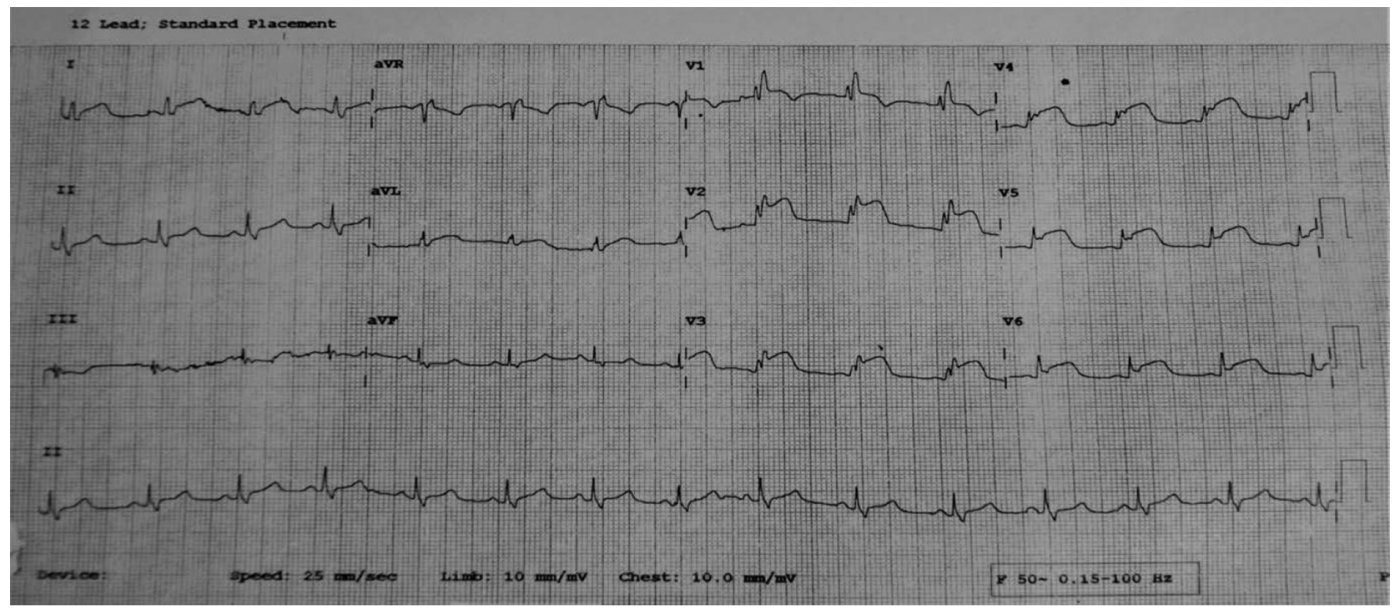

Fig. 2 2nd ECG on presentation: AWMI. Abbreviation: AWMI, anterior wall myocardial infarction.

Her parents were afflicted with hypertension (HTN), and her mother had h/o two spontaneous abortions, with no h/o any major cardiac events in family members.

She experienced emotional stress regarding her personal issues for the last 1 year.

She was hemodynamically stable. Her general and systemic examination was normal, except obesity with body mass index (BMI) of $32 \mathrm{Kg} / \mathrm{m} 2$.

Primary treatment was given and the patient was shifted to the Cath-laboratory for primary percutaneous coronary intervention (PCI) (radial approach) with door to balloon time being $<90$ minutes ( 80 minutes).

Coronary angiography (CAG) showed total thrombotic occlusion of LAD from midsegment, thrombotic occlusion of 1 st septal and diagonal branch, with some thrombus also noted in ostioproximal LAD. ( - Fig. 3 ) Left circumflex (LCX) artery was normal, ramus was normal, and right coronary artery (RCA) was dominant and normal.

Manual aspiration of thrombus was done ( - Fig. 4), and stenting was deferred as stenosis was less than $50 \%$.

-Fig. 5 represent results of postthrombosuction.

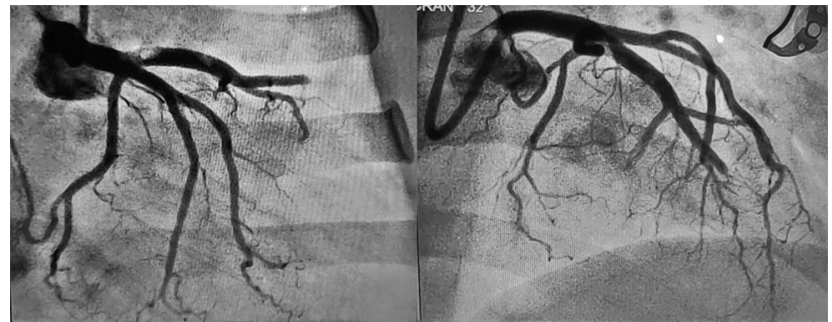

Fig. 3 Total thrombotic occlusion of LAD artery in RAO caudal and LAO cranial views. Abbreviations: LAD, left anterior descending; LAO, left anterior oblique; RAO, right anterior oblique.
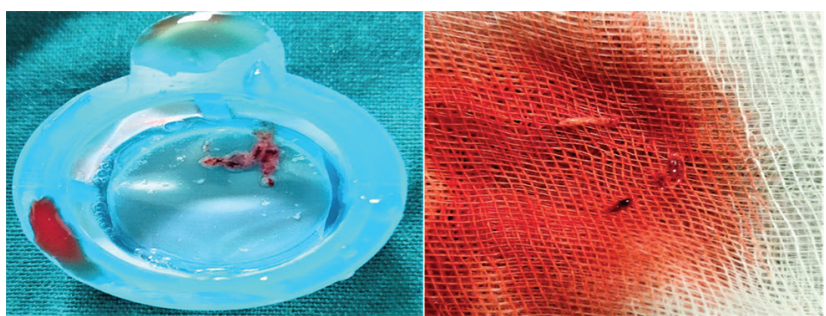

Fig. 4 Aspirated thrombus. 


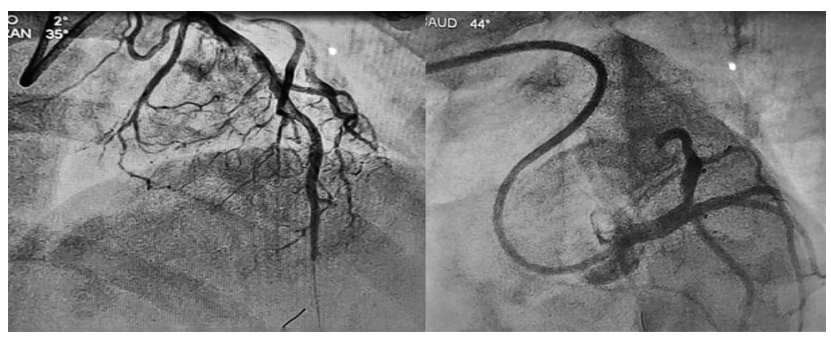

Fig. 5 Postthrombosuction: AP cranial and LAO caudal views showing decreased thrombotic burden with insignificant residual stenosis in LAD. Abbreviations: LAD, left anterior descending; LAO, left anterior oblique.

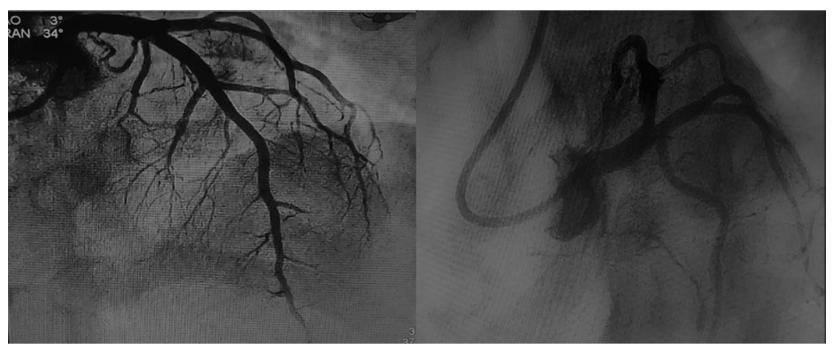

Fig. 7 Post-PCI final result (after 24 hours of Tirofiban infusion), AP cranial and LAO caudal views showing TIMI grade 3 flow with insignificant residual stenosis in $\mathrm{LAD}$. Abbreviations: $\mathrm{LAD}$, left anterior descending; $\mathrm{LAO}$, left anterior oblique; TIMI, thrombolysis in myocardial infarction.

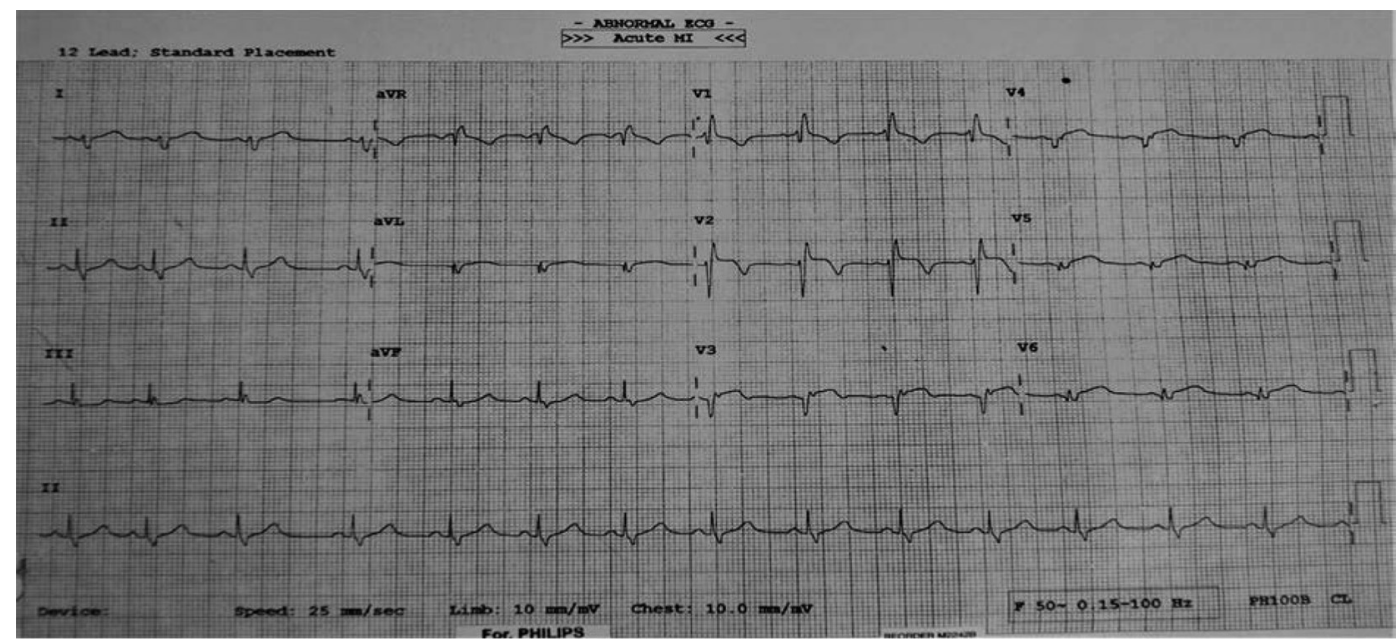

Fig. 6 Post-PCI ECG: Almost isoelectric ST segment in anterior leads.

Post-PCI, patient was hemodynamically stable and shifted to CCU for further care. Post-PCI ECG revealed almost isoelectric ST segment without any chest discomfort (-Fig. 6). Along with routine post-PCI regimen, patient was started on Tirofiban infusion in standard dose for 24 hours, and check shoot angiography after 24 hours revealed TIMI grade 3 flow with residual insignificant stenosis. ( - Fig. 7)

The patient's hemogram was normal, except WBC: 17,200/microL. Her CPK-MB was 258 and TROP- I was 47,605 . Her lipid profile, TFT, and CXR were normal. Serum B12 was $148 \mathrm{pg} / \mathrm{L}$ (lower limit of normal range), homocystein was 57 microgram/L (elevated > 15), folic acid was 4.3 (normal), and antinuclear antibody (ANA) blot assay: Negative and coagulation profile revealed isolated protein C deficiency.

Patient was hemodynamically stable at the time of discharge. The patient was regularly attending follow-up and doing well on medications. Follow-up ECHO showed EF 60\%

The patient was having anterior wall STEMI with risk factors, PCOS, OC pills, obesity (BMI $>32 \mathrm{~kg} / \mathrm{m} 2$ ), hyperhomocysteinemia, isolated protein $\mathrm{C}$ deficiency, emotional stress, and endothelial dysfunction.

\section{Discussion}

Risk factors for MI in young patients include traditional risk factors for atherosclerosis and other factors such as $\mathrm{F} / \mathrm{H} / \mathrm{O}$ premature coronary artery disease (CAD), coronary embolism, coronary spasm, coronary dissection, coronary aneurysm, coronary vasculitis, collagen vascular diseases, hypercoagulability states, drugs, and stress.

Unique and novel risk factors that occur more commonly or exclusively in women are preeclampsia, eclampsia, pregnancy-induced hypertension (PIH), gestational diabetes, systemic lupus erythematosus (SLE), rheumatoid arthritis (RA), and psychosocial: anxiety, depression, job-marital stress..$^{-3}$

PCOS is associated with increased risk of DM, HTN, and dyslipidemia causing endothelial dysfunction. ${ }^{4-6}$ Hyperandrogenism (free testosterone) in PCOS is associated with systolic blood pressure ${ }^{7}$ and abnormal lipid metabolism. ${ }^{8}$

If traditional risk factors are present in women taking OCs, then underlying atherosclerosis process is augmented, subsequently increasing the risk of ACS at a younger age. ${ }^{9}$

Estrogen-containing pills are more prone for the development of thrombotic events, both arterial and venous, than 
progesterone-containing pills. However, there are some studies showing increased thrombotic risk with the use of progesterone-only pills for longer duration of time (in years) by unknown mechanisms. In our case, the patient was taking tablet DEVIRY $10 \mathrm{mg}$ (medroxyprogesterone) for 3 to 4 years.

Even a low-dose OC for a shorter period of time even without any risk factors for CAD can cause AMI but incidence is rare. ${ }^{10}$ Therefore, treating physicians as well as patients on OCs must be aware of signs, symptoms and risk factors of CAD. Care must be taken to avoid, at least, modifiable risk factors for MI. In our case, the patient was taking tablet ORAL-L (0.15/0.03 $\mathrm{mg}$ ) since the last 1 month.

Isolated protein $\mathrm{C}$ deficiency can occur in patients taking OCPs for a longer duration of time. ${ }^{11}$

Serum homocysteine can be elevated in some patients of PCOS and also be associated with increased risk of vascular thromboembolism. ${ }^{12}$

Endothelium is the inner most lining of blood vessel (Tunica intima) by monolayer of endothelial cells. It lines the entire circulatory system: from heart to capillaries. ${ }^{13}$ The functions of endothelium are as follows: acts as a physical barrier between the blood and vessel wall, transports nutrients between blood and tissues, and serves anti-inflammatory, antithrombotic and vasoregulatory purposes. Normal endothelial function is characterized by a fine balance between vasoconstrictors and vasodilators, inflammatory and anti-inflammatory factors, and thrombotic and antithrombotic factors. ${ }^{14-16}$

Endothelial dysfunction occurs as a result of decreased vasodilators (NO), increased vasoconstrictors, increased oxidative damage, increased inflammatory factors, increased thrombotic factors, and increased atherosclerotic factors, ultimately causing thrombotic occlusion of vessel following atherosclerotic plaque rupture/ erosion. ${ }^{14-16}$

\section{Conclusion}

Incidence of CAD is increasing among those $<40$ years. Detailed history, thorough clinical examination along with relevant investigations helps us to detect early diagnosis of AMI. But above all, suspicion regarding ACS is the most important factor in managing these cases. Most of the young patients have atherosclerotic plaque erosion $\mathrm{f} / \mathrm{b}$ thrombotic occlusion of culprit vessel and have a tendency toward complete recanalization without need of coronary stenting. Along with optimal medical treatment, identification and treatment for modifiable risk factors is also mandatory.

In symptomatic women with angina, despite the absence of obstructive stenosis on CAG, one must consider and further investigate coronary microvascular dysfunction, endothelial dysfunction, microvascular spasm, spontaneous coronary artery dissection, and other nonvascular myocardial causes.

\section{Conflict of Interest}

None.

\section{References}

1 Mosca L, Benjamin EJ, Berra K, et al. Effectiveness-based guidelines for the prevention of cardiovascular disease in women2011 update: a guideline from the american heart association. Circulation 2011;123(11):1243-1262

2 Al Mheid I, Quyyumi AA. Sex differences in mental stress-induced myocardial ischemia: are women from venus? J Am Coll Cardiol 2014;64(16):1679-1680

3 Shah AJ, Ghasemzadeh N, Zaragoza-Macias E, et al. Sex and age differences in the association of depression with obstructive coronary artery disease and adverse cardiovascular events. J Am Heart Assoc 2014;3(3):e000741

4 Lo JC, Feigenbaum SL, Yang J, Pressman AR, Selby JV, Go AS. Epidemiology and adverse cardiovascular risk profile of diagnosed polycystic ovary syndrome. J Clin Endocrinol Metab 2006;91(4):1357-1363

5 Talbott E, Clerici A, Berga SL, et al. Adverse lipid and coronary heart disease risk profiles in young women with polycystic ovary syndrome: results of a case-control study. J Clin Epidemiol 1998;51(5):415-422

6 Tarkun I, Arslan BC, Cantürk Z, Türemen E, Sahin T, Duman C. Endothelial dysfunction in young women with polycystic ovary syndrome: relationship with insulin resistance and low-grade chronic inflammation. J Clin Endocrinol Metab 2004;89(11):5592-5596

7 Phillips GB, Pinkernell BH, Jing TY. Relationship between serum sex hormones and coronary artery disease in postmenopausal women. Arterioscler Thromb Vasc Biol 1997;17(4):695-701

8 Wu FCW, von Eckardstein A. Androgens and coronary artery disease. Endocr Rev 2003;24(2):183-217

9 Talbott E, Guzick D, Clerici A, et al. Coronary heart disease risk factors in women with polycystic ovary syndrome. Arterioscler Thromb Vasc Biol 1995;15(7):821-826

10 Aslan AN, Süygün H, Sivri S, Keleş T. Low-dose oral contraceptive-induced acute myocardial infarction. Eur J Contracept Reprod Health Care 2016;21(6):499-501

11 Wagh SB, Anadure R, Dutta V, Sandhu MS, Trehan R. Isolated protein $\mathrm{S}$ deficiency presenting as catastrophic systemic arterial and subsequently venous thrombosis. Australas Med J 2012;5(8):424-428

12 Yarali H, Yildirir A, Aybar F, et al. Diastolic dysfunction and increased serum homocysteine concentrations may contribute to increased cardiovascular risk in patients with polycystic ovary syndrome. Fertil Steril 2001;76(3):511-516

13 Limaya V, Vadas $M$, The vascular endothelium: structure and function. In: Fitridge R, Thompson M, eds. Mechanisms of Vascular Disease A TEXTBOOK FOR Vascular Surgeons. 1st ed. Cambridge University Press. 2007;1-14

14 Esper RJ, Nordaby RA, Vilariño JO, Paragano A, Cacharrón JL, Machado RA. Endothelial dysfunction: a comprehensive appraisal. Cardiovasc Diabetol 2006;5:4

15 Oufella AH, Maury E, Lehoux S, et al. The endothelium: physiological functions and role in microcirculatory failure during severe sepsis. Intensive Care Med (e-pub ahead of print). doi: 10.1007/s000011134-010-1893-6

16 Vita JA. Endothelial function. Circulation 2011;124(25): e906-e912 